\title{
REVISIONES
}

\section{La promoción de experiencias de innovación educativa en TIC en los centros escolares. Caso región Caribe colombiana*}

\author{
The promotion of educational innovation of ICT in schools. \\ The case of the Caribbean region of Colombia
}

\author{
Elias Said-Hung, ${ }^{a}$ Jorge Valencia-Cobos,${ }^{b}$ Evaristo González Prieto ${ }^{c}$ \\ a'Universidad Internacional de la Rioja \\ Telf.: (34) 692959503. Correo electrónico: esaidh@ gmail.com \\ bUniversidad del Norte \\ Telf.: (57) 53509509. Correo electrónico: javalenciac@uninorte.edu.co \\ 'Torre del Palau, España \\ Telf.: (34) 619692955. Correo electrónico: evaristogonzal@ gmail.com
}

\begin{abstract}
El presente artículo busca analizar los factores vinculados al contexto institucional de los centros escolares oficiales, los cuales han contribuido a la promoción de experiencias de innovación educativa mediadas por TIC. Ello, a partir del análisis de los datos recolectados en un proyecto enmarcado dentro del paradigma empíricopositivista, donde se aplicó una encuesta, diligenciada por 172 directores docentes o rectores de instituciones de educación oficial, $1-\alpha=95 \%$ y ee $= \pm 5 \%$, representativas de la región Caribe colombiana. Los resultados nos ayudan estimar el perfil que tienen las instituciones innovadoras en el área TIC, así como también la percepción institucional alrededor del aprovechamiento de estas y las estrategias de integración pedagógicas puestas en marcha por este tipo de entidades, en comparación con las no tipificadas como tales; y los factores que inciden en la promoción de instituciones con capacidad de generación de experiencias educativas innovadoras, mediadas por los recursos y dispositivos tecnológicos.
\end{abstract}

Palabras clave: innovación educativa, tecnología de la información, escuelas, sistema educativo.

\begin{abstract}
This paper analyzes the factors related to the institutional context of official schools, which have contributed to the promotion of innovative educational experiences mediated by ICT. This information emerged from the analysis of data collected in a project framed within the empirical-positivist paradigm, through a survey filled out by 172 rectors of education institutions, $1-\alpha=95 \%$ and applied ee $= \pm 5 \%$, representative of the Colombian Caribbean region. The results help us to estimate the profile of innovative institutions in the ICT area; the institutional perception about the use of these teaching strategies and those of integration carried out by such institutions, when compared to those non-classified as such; and the factors that influence the development of institutions with capacity to generate innovative educational experiences, mediated by resources and technological devices.

Key words: education innovation, information technology, schools, educational systems.
\end{abstract}




\section{INTRODUCCIÓN}

La educación actual se halla inmersa en un proceso de adaptación a los cambios motivados por las nuevas demandas de formación de una sociedad global en constante evolución, donde los avances tecnológicos provocan reformulaciones de las metodologías tradicionales de la enseñanza y nuevas apuestas, en las que la innovación ocupa un lugar predominante.

Autores como Fariña y Sosa (2011), Claro et al. (2012), Arias y Cristia (2014) y Ait et al. (2015), entre otros, han venido analizando, desde diferentes perspectivas, los diversos factores que inciden al momento de promover la integración de las Tecnologías de Información y las Comunicaciones (TIC) en los procesos de enseñanza y aprendizaje; así como en el uso que se está haciendo de los diferentes recursos y dispositivos tecnológicos en los contextos escolares y en el fomento de competencias del siglo XXI. Algunos de los avances hechos en los estudios realizados por estos autores apuntan a los docentes, al clima escolar, la gestión de las TIC, así como al rol que tienen los profesionales de la educación y coordinadores académicos en la integración de estas y la formación del profesorado, en favor de la integración pedagógica de las tecnologías, a través de los contenidos curriculares y métodos evaluativos adaptados.

A partir de estudios realizados hasta la fecha, se han logrado además identificar un conjunto de factores que traen consigo el aumento del proceso de integración de las TIC en los centros escolares o instituciones educativas. Tal es el caso de expuesto por autores como Ananiadou y Claro (2009) y Area (2010), quienes han destacado la importancia que tienen las innovaciones llevadas a la práctica, tanto a nivel organizativo como en el desarrollo profesional de los docentes y en la enseñanza aplicada a los estudiantes por parte de los profesores y demás miembros responsables de la formación de estos; así como del proceso de aprendizaje llevado a cabo por los propios alumnos.

En la actualidad, la labor que tienen las instituciones educativas, en aras de favorecer el proceso de integración de las TIC, debe centrarse en la reestructuración del entorno institucional que favorezca el cambio en las estrategias y métodos de enseñanza-aprendizaje aplicados hasta ahora, con el fin de garantizar en sus estudiantes el máximo aprovechamiento de las TIC para afrontar exitosamente los retos que deberán superar en las sociedades contemporáneas actuales, caracterizadas por la globalidad y por la primacía de los entornos digitales. Para ello, es necesario asumir una postura en torno a las TIC, más allá de destacar el impacto directo, per se, que puedan traer consigo los recursos y dispositivos tecnológicos. También se precisa ahondar en cómo sacar el máximo provecho, tanto a nivel comunicativo como educativo, del conjunto de la tecnología dispuesta en la actualidad (Claro, 2010; Papanastasiou \& Ferdig, 2006). Por ello, en la medida en que los centros educativos cuenten con procesos de enseñanzaaprendizaje adaptados y con capacidad de dar respuesta a los retos de nuestras sociedades contemporáneas, inmersas en lo que se ha dado en llamar Sociedad de la Información y el Conocimiento (SIC), se podrá avanzar en el establecimiento de nuevas habilidades y competencias, orientadas a garantizar el acceso y uso efectivo de la información, así como en la mejora de la gestión del conocimiento, por citar algunos aspectos relevantes a la fecha (Cox et al., 2003).

Para lograr la transformación del entorno educativo, en general, estimamos que se necesita garantizar la incorporación de las TIC, no solo en los procesos pedagógicos y educativos, sino también en los procesos administrativos, de gestión académica y comunicativa, llevados a cabo en el interior de las instituciones educativas. Esto, con el propósito de garantizar la transformación de este entorno hacia el aprendizaje colectivo de todos los miembros que 
hacen parte de él. Hecho que implicaría la transformación de la cultura impuesta en los centros de enseñanza, por medio del acceso y uso significativo (tanto desde el punto de vista de la comunicación como también educativo) de los diferentes recursos y dispositivos tecnológicos dispuestos en la actualidad (Fullan, 2007); convirtiéndose así la escuela en un nodo importante de promoción de la SIC.

Autores como Ling, Woo y Lim (2013) han destacado que la integración de las TIC en los escenarios de enseñanza-aprendizaje requiere de una planificación estratégica, orientada a una mayor articulación de estas en los currículos, como en la oportuna contextualización de este proceso desde la propia realidad de cada institución y la formación de sus docentes y demás actores educativos (rectores y coordinadores académicos, por ejemplo). Ello, desde una perspectiva donde se reconozcan las potencialidades que pueden traer consigo las TIC en el desarrollo de la vida escolar, en especial a nivel pedagógico (Arias \& Crista, 2014; Benavides \& Pedró, 2007). Para los fines aquí señalados, se requiere tener en cuenta lo expuesto por autores como García-Valcárcel y Tejedor (2011) en cuanto al necesario cambio en la forma en como es concebida la enseñanza, el aprendizaje y los proyectos educativos que rigen el quehacer de los docentes. Hecho que lleva a replantear las metodologías de enseñanza tradicionales, ante la inclusión de nuevos tipos de actividades y recursos digitales, dispuestos en la actualidad para el aprendizaje.

\section{EL ROL DE LOS AGENTES EDUCATIVOS EN LA INNOVACIÓN EN TIC EN LOS CENTROS ESCOLARES}

Como expusimos en el apartado anterior, el contexto educativo actual se encuentra en pleno proceso de adaptación sobre cómo se lleva a cabo la inclusión de las TIC en su interior. Es en el marco de lo aquí expuesto que los integrantes del conjunto de actores educativos (directivos docentes o rectores, coordinadores académicos, docentes, estudiantes y comunidad en general) deben ser reconocidos como agentes que contribuyen a la potencial transformación del entorno institucional y pedagógico requerido para garantizar un mayor nivel de aprovechamiento de los dispositivos y recursos tecnológicos actuales (Boza, Tirado \& Guzmán-Franco, 2010; Said-Hung et al., 2015).

Los retos que tiene la educación apuntan a los señalamientos dados por autores como Domingo-Coscollola y Marquès-Graells (2011), Binkley et al. (2012), Sunkel, Trucco y Espejo (2013) y la Unesco (2013), en especial en la necesidad de tener presente los efectos que producen las TIC en el aula en relación con la generación de nuevos procesos de aprendizaje y conocimiento, así como en la producción de nuevos contextos de innovación pedagógica (en la creación, diseño, intercambio, publicación, adaptación y producción desde internet y las Web 2.0).

Como bien han venido destacando autores como Craig, Ault y Niileksela (2011), Sang et al. (2011), Fariña y Sosa (2011), Ramírez, Cañedo y Clemente (2012), Area, Gutiérrez y Vidal (2012), Arias y Cristia, 2014 o Said-Hung et al. (2015), para que cualquier proyecto educativo resulte exitoso se requiere del compromiso y actitud positiva no solo de los docentes, quienes pueden aducir explicaciones (muchas veces plasmadas en estudios realizados por los autores antes mencionados) sobre el bajo nivel de aprovechamiento de las TIC, la falta de voluntad pedagógica, la inhabilidad en el empleo de estas o bien causas externas a ellos (por ejemplo el estado de la infraestructura y el apoyo institucional). 
En general, los factores que inciden en la integración de las TIC en los escenarios educativos dependen de mecanismos eficaces que ayuden a trascender el discurso de la innovación, a través del diseño de programas, experiencias educativas significativas y estrategias pedagógicas que guarden relación directa con la realidad existente en el interior de cada escenario de enseñanza (Montero \& Gewerc, 2010). Lo aquí mencionado apunta a destacar la necesidad de llevar a cabo acciones orientadas a conseguir el uso intensivo de las TIC, no solo desde el punto de vista meramente técnico o desde la infraestructura, sino desde el empleo comunicativo y educativo de estas, en el interior de los espacios institucionales empleados para la formación integral de los estudiantes. Esto, desde la ejecución de diferentes actividades pedagógicas, lideradas por docentes y demás miembros de la comunidad educativa con sus estudiantes, sobre la base de que lo importante es lograr incidir en el cómo y para qué se usan las TIC (aspectos metodológicos), a favor del desarrollo de habilidades y competencias del siglo XXI, más que en la simple dotación de equipos tecnológicos en el aula o espacios educativos institucionales.

Bajo el escenario descrito hasta ahora, el rol que asumen docentes, coordinadores académicos y directivos, en el interior de los escenarios de enseñanza-aprendizaje, resulta clave en la planificación de dichos entornos (Vera, 2004). Ello desde la concepción asumida en este trabajo: si bien el docente juega un importante papel en la formación de los estudiantes, no significa que el resto de actores (e.g. directivos) no adquieran importancia en el momento de fomentar un contexto favorable para el desarrollo profesional y aplicación de las diferentes actividades formativas a cargo de estos.

Como bien destacan Said-Hung et al. (2015), gestores educativos como los directivos docentes (rectores) son los máximos líderes en el desarrollo de los procesos administrativos, académicos, pedagógicos y técnicos llevados a cabo en el interior de las instituciones educativas. Están a cargo de la cultura institucional requerida para el desarrollo de las diversas funciones desempeñadas por todos los actores vinculados a este contexto, así como también tienen la responsabilidad de canalizar las diversas tensiones generadas entre los objetivos e intereses colectivos e individuales de sus miembros, además de garantizar la consecución de los resultados de calidad del aprendizaje brindado a sus alumnos y favorecer la promoción de un entorno donde se promueva el uso de las TIC, entre otros aspectos que aluden a la organización global de la escuela y de todo su accionar interno. Por ello, ahondado en torno a las características del liderazgo y reconociendo el compromiso asumido por gestores educativos como los directivos docentes y coordinadores académicos, resulta clave su papel para definir el devenir de cada una de las instituciones educativas a su cargo (Beraún, 2011).

En el caso de los docentes, uno de los retos que estos tienen ante sí en la Era Digital es la capacidad de afrontar los rápidos cambios que está trayendo consigo el avance de las TIC en todos los contextos de nuestras sociedades contemporáneas, en nuestro caso en los escenarios escolares actuales. Ello, desde un entorno cada vez más orientado a hacer que los docentes no asuman el rol tradicional dentro del proceso de aprendizaje de sus estudiantes (responsables principales del aprendizaje en el aula), sino que tiendan a ejercer más una función de guía en la enseñanza-aprendizaje (Daniels, 2015).

Por todo lo aquí expuesto, la implicación que tiene la totalidad de actores vinculados a las instituciones educativas tiende a un efecto inmediato en el cambio del rol asumido por los docentes, ya que desde la práctica de la gestión educativa, el docente interesado en sacar máximo aprovechamiento educativo de las TIC ha de promover el sentido de la curiosidad 
alrededor de nuevas metodologías y prácticas educativas novedosas; la actualización a favor de su desarrollo profesional como docentes, para sacar máximo provecho a las potencialidades inherentes a las tecnologías; la mentalidad abierta y flexible, requerida para la adaptación a los cambios generados a partir de los avances de las TIC; la colaboración entre pares y otros agentes educativos, favoreciendo la sinergia y aprendizaje colectivo; y el liderazgo "horizontal" requerido para el máximo aprovechamiento del conocimiento obtenido a través del uso de los diferentes recursos y dispositivos tecnológicos actuales, entre otros aspectos.

Nuestro presente exige debatir el rol asumido por los escenarios de enseñanza alrededor de la necesidad de mejorar el desempeño de las diferentes habilidades y competencias presentes en los estudiantes bajo la mediación de los avances tecnológicos (Vanderlinde \& Van Braak, 2011), ya que nos encontramos en la actualidad inmersos en contextos educativos donde parecieran coexistir, de forma no lineal, en medio de los diferentes momentos indicados por la IIPE-UNESCO (2006), a saber: momento pre-pc, donde el debate se centraba en la pertinencia en la formación básica de programación y el aprendizaje por descubrimiento; momento informático, donde el debate gira más en la generación de escenarios formativos orientados a la alfabetización digital desde las escuelas; momento internet, donde el debate se enmarca en el proceso de inclusión de dispositivos tecnológicos en los escenarios de enseñanza y el fomento de capacitaciones dirigidas a los docentes; y momento actual, donde el debate se centra más en los procesos de aprendizaje colaborativo y colectivo, y la búsqueda de mecanismos que aseguren la integración de los usos pedagógicos y administrativos de las TIC. Es por ello que resulta conveniente ahondar en el análisis que permita no solo identificar los rasgos que caracterizan a las instituciones educativas que han asumido el liderazgo de promoción de experiencias innovación educativa mediadas por TIC, sino también avanzar en el proceso de identificación de factores que contribuyen a tales fines para el establecimiento de algunas líneas de acción que ayuden a la integración efectiva de la tecnología a nivel del conjunto de instituciones educativas dispuestas en la actualidad en nuestras sociedades contemporáneas.

\section{METODOLOGÍA}

El objetivo del presente artículo es analizar los factores vinculados al contexto institucional de los centros escolares oficiales (escuelas públicas) que han contribuido a la generación de experiencias de innovación educativa, bajo la mediación de las TIC. Ello, a partir de los resultados generales obtenidos en el marco de un proyecto de investigación enmarcado dentro del paradigma empírico-positivista, donde se tomó como la población de estudio todas las instituciones educativas oficiales adscritas a las secretarías de educación de la región Caribe de Colombia, conformada por 7 Departamentos o unidades territoriales (32 Departamentos tiene Colombia, en total), los cuales cuentan con un total de 194 municipios, 22 de los cuales son considerados como Entidades Territoriales Certificadas (ETC), es decir, cuentan con autonomía administrativa, al momento de dirigir, planificar, organizar y prestar el servicio educativo en los niveles de preescolar, básica (primaria) y media (secundaria o bachillerato). Del total de ETC dispuestas a nivel de la región Caribe de Colombia, se tomó como área geográfica de aplicación del trabajo de campo al departamento del Atlántico. La selección de este Departamento se hizo sobre la base de los siguientes criterios: 
- $\quad$ Representa el $21 \%$ de la población en edad escolar.

- Concentran el $24 \%$ de los establecimientos educativos.

- $\quad$ Concentra el 20\% de los estudiantes matriculados en la Región Caribe.

- Los indicadores de infraestructura TIC y conectividad son más elevados en comparación con los otros departamentos de la Región.

- $\quad$ Representa el 26\% del PIB Regional (a precios constante por departamentos Base 2005).

En cuanto al diseño muestral se desarrolló un diseño aleatorio estratificado, de acuerdo con el estrato socio-económico de la ETC, la zona (rural y urbana) y las sedes de las instituciones educativas donde se aplicó el instrumento de medición (encuesta) pautado en el marco del proyecto en el que se basó este trabajo. La muestra final contó con afijación proporcional, compuesta por 172 instituciones educativas representativas del universo poblacional con $1-\alpha=95 \%$ y ee $= \pm 5 \%$.

Las encuestas llevadas a cabo fueron aplicadas a directivos docentes (rectores) de las instituciones educativas tomadas como unidades muestrales, ya que son los máximos responsables al momento de dirigir y marcar las pautas de desarrollo de las diferentes funciones, tanto a nivel administrativo como educativo, al interior de los escenarios de enseñanza. El instrumento empleado para la recolección de los datos expuestos en este trabajo fue validado a través de una prueba piloto, orientada a garantizar la validez y confiabilidad de la encuesta final aplicada. Para los fines aquí indicados, se empleó el coeficiente de Alfa de Cronbach, definido como un índice usado para medir la confiabilidad del tipo consistencia interna de una escala, es decir, para evaluar la magnitud en que los ítems de un instrumento están correlacionados (Oviedo \& Campo, 2005). Así, pues, los valores más altos del Alfa de Cronbach serán indicadores de mayores niveles de relación y, por ende, mayor consistencia (Tabla 1).

Tabla 1. Estadísticos de fiabilidad en bloques de preguntas que integraban la encuesta aplicada a directivos

\begin{tabular}{|c|c|}
\hline \multicolumn{2}{|c|}{ Preguntas relacionadas con la disponibilidad TIC } \\
\hline Alfa de Cronbach & N de elementos \\
\hline 0,865 & 14 \\
\hline $\begin{array}{r}\text { Preguntas relacionadas con la organización de la institución } \\
\text { educativa alrededor de las TIC }\end{array}$ \\
\hline Alfa de Cronbach & N de elementos \\
\hline 0,832 & 29 \\
\hline Preguntas relacionadas con la formación y competencia de los \\
docentes en el uso de las TIC \\
\hline Alfa de Cronbach & N de elementos \\
\hline 0,902 & 28 \\
\hline
\end{tabular}




\begin{tabular}{|c|c|}
\hline \multicolumn{2}{|c|}{ Preguntas relacionadas con la actitud de los docentes ante las TIC } \\
\hline Alfa de Cronbach & N de elementos \\
\hline 0,911 & 24 \\
\hline
\end{tabular}

Nota: $\mathrm{n}=172$ instituciones educativas oficiales.

La recolección de información se realizó durante los meses de septiembre y diciembre de 2013. Concluido el proceso de recolección, verificación y depuración de los datos obtenidos, se realizaron los diferentes análisis multivariados expuestos en este artículo, a través del software IBM SPSS Satistics 20.

Para el estudio del tema propuesto, se tomó como variable dependiente la existencia de una o más experiencias TIC registradas y sistematizadas al interior de las instituciones educativas analizadas, considerando que este tipo de actividades, además de sus alcances cognitivos, propicia la mejora de la propia práctica y cualifica a sus actores, además de generar ajustes, desplazamientos y cambios necesarios para que el programa o proyecto sistematizado gane en eficacia social y riqueza cultural (Peñafiel, 2000). Desde el criterio de que la existencia formal de experiencias significativas o innovadoras en materia educativa en la muestra de casos seleccionada mediadas por las TIC, termina siendo una medida oficial y tangible del tema aquí propuesto. A partir de esta variable, hemos categorizado las instituciones analizadas en dos tipos: instituciones innovadoras, con experiencias TIC registradas y sistematizadas, e instituciones no innovadoras, sin experiencia registrada a la fecha de levantamiento de la información tomada para el abordaje del tema propuesto.

\section{RESULTADOS}

\subsection{PERFIL DE LAS INSTITUCIONES INNOVADORAS EN TIC}

En términos generales, observamos cómo el 39,9\% de las instituciones educativas participantes en el estudio $(n=172)$ dieron cuenta o contaban con alguna experiencia TIC registrada y sistematizada en el momento de desarrollo de este proyecto. Hecho que nos permite ver el bajo nivel de porcentaje de innovación en TIC aplicado, al menos al interior de nuestra muestra.

Los datos recolectados nos permiten establecer un conjunto de rasgos generales que caracterizan a la mayoría de las instituciones educativas que pudiésemos considerar innovadoras en materia TIC, al llevar a cabo acciones a su interior donde se da cuenta clara de un ambiente escolar orientado al aprovechamiento educativo de diferentes recursos y dispositivos tecnológicos:

- La relación profesor por alumno es menor, grupos más pequeños por docente, en las instituciones educativas oficiales con experiencias innovadores en TIC, que en aquellas instituciones que no cuentan con experiencias de este tipo $(n=172)$. En el primer caso, la media $(\mu)$ de alumnos por docentes fue de 27 , mientras que en el segundo grupo fue de 30 . 
- Un mayor porcentaje de este tipo de instituciones $(\mathrm{n}=143$ y casos perdidos=29) han orientado la formación de sus docentes en el uso de las TIC $(91,7 \%)$, en comparación con las no tipificadas $(69,5 \%)$, es decir, 22,2 puntos porcentuales a favor del primer grupo.

- Un mayor porcentaje de estas instituciones ( $\mathrm{n}=137$ y casos perdidos=35) cuentan con proyectos orientados a ayudar a los miembros de la comunidad educativa a la adquisición de habilidades de organización comprensión, evaluación y análisis de información a través de las TIC (80\%), en comparación con los casos tipificados como no innovadoras $(34,5 \%)$.

- $\quad$ Presentan una mayor diversificación de las fuentes de financiación empleada para la dotación de equipamiento tecnológico, en comparación con las instituciones no innovadoras. Dentro de la muestra analizada $(\mathrm{n}=169$ y casos perdidos $=3)$, si contrastamos ambos grupos, se puede observar un mayor porcentaje de fondos o recursos propios (28,8\% versus $20 \%)$, así como mayores niveles de participación de la comunidad $(1,7 \%$ versus $0 \%)$ y de fondos provenientes de diferentes instancias públicas, no solo a nivel Estatal (47,5\% versus $67,5 \%)$, sino también a nivel departamental (13,6\% versus $5,5 \%)$ y municipal (8,5\% versus $6,4 \%)$.

- Existe un mayor porcentaje de instituciones innovadoras en TIC en zonas urbanas que rurales. En el conjunto de instituciones educativas oficiales analizadas $(n=172)$, si comparamos este grupo de instituciones con aquellas no tipificadas como innovadoras, observamos cómo la diferencia entre ambos grupos por zonas, respectivamente, es de 4.2 puntos porcentuales menos ( $84,7 \%$ versus $80,5 \%)$ y 4 puntos porcentuales más (11,9\% versus $15,9 \%)$.

\subsection{PERCEPCIÓN INSTITUCIONAL SOBRE LAS TIC Y ESTRATEGIAS DE INTEGRACIÓN}

Los datos obtenidos a partir de las respuestas dadas por los directivos docentes de las 172 instituciones educativas oficiales encuestadas nos permite observar cómo, a nivel de percepción sobre las TIC, el escenario institucional estudiado, en términos generales, está caracterizado por presentar un porcentaje alto de percepción favorable sobre la utilidad de las TIC a nivel educativo y gestión institucional, tanto en el grupo de instituciones tipificadas como innovadoras como las que no se ubican dentro de esta categoría. En otras palabras, en la mayoría de los casos (por encima del 85\%), los directivos docentes (rectores) encuestados se mostraron de acuerdo a considerar que las TIC han contribuido, de forma alta, a mejorar los procesos de comunicación, en los procesos de enseñanza y calidad educativa impartida, así como en los procesos administrativos llevados a cabo en sus instituciones.

En lo que se refiere a la percepción observada sobre el uso de las TIC en la gestión académica, al interior de las 172 instituciones educativas analizadas, los datos recolectados nos muestran un entorno de alta (total o parcialmente favorable) percepción, tanto en las instituciones innovadoras como las que no se ubican en esta categoría, con relación a

- El impacto de las TIC en actividades vinculadas con la matrícula y seguimiento del rendimiento académico de los estudiantes (más del 85\%).

- En la comunicación e interacción con los docentes (más del 95\%), las Secretarías de Educación, ente público responsable del cumplimiento de las labores 
institucionales relacionadas con los escenarios de enseñanza analizados (más del $86 \%$ ) y con otras entidades afines (más del 80\%).

- $\quad$ En la planeación de la institución (más del 90\%), los procedimientos contables y financieros llevados a cabo por los escenarios escolares analizados (más del 85\%), la evaluación institucional (más del 85\%) y en la evaluación de los docentes (más del $80 \%)$.

- En el delineo de los programas de enseñanza-aprendizaje impartidos por los docentes vinculados con las instituciones analizadas (85\%) y en el fortalecimiento de la enseñanza-aprendizaje basada por competencia en los escenarios de enseñanza (más del 90\%).

- En la producción de información y fomento del pensamiento crítico y la reflexión de los alumnos matriculados en el aula (más del 75\%).

Donde menor porcentaje de encuestados reconocieron las potencialidades del uso de las TIC en la gestión académica, a nivel general, fue en el establecimiento de comunicación con los padres y madres de familia o responsables legales de sus estudiantes. Pese a que los niveles de percepción favorable (total o parciales) se ubicaron sobre el 50\% del total de casos analizados ( las instituciones innovadoras son las que se observaron un mayor porcentaje de directivos docentes que manifestaron no hacer uso de los recursos y dispositivos tecnológicos o la ausencia de cambio alrededor del tema aquí expuesto (47\%). Ello, en comparación a las instituciones consideradas como innovadoras en este trabajo, donde el $34 \%$ de los directivos manifestaron lo antes expuesto. Es decir, en el grupo de instituciones no innovadoras, la falta de uso o falta de impacto de las TIC en la promoción de canales que contribuyan a una mayor vinculación y/o participación de los padres y/o madres o representantes legales en todo lo relacionado con la formación académica de sus estudiantes fue mayor, aunque igualmente alta, que en el caso de las instituciones consideradas aquí como innovadoras.

En cuanto a las percepciones que tenían los directivos de las instituciones encuestadas en relación al interés observado de sus docentes en uso de las TIC, los datos nos permiten apreciar cómo, en términos generales, la percepción fue muy favorable (más del 68\%) en la totalidad de las instituciones analizadas (sin importar si eran o no innovadoras), en torno al uso educativo de las TIC en los profesores jóvenes; el interés mostrado por los profesores en la formación en el uso educativo de recursos y dispositivos tecnológicos; y en el apoyo de los docentes en el proceso de la integración de las TIC en los proyectos educativos institucionales y el apoyo de los docentes, a favor de la mejora de la calidad educativa impartida en los centros escolares. Así mismo, se aprecia también cómo la percepción en torno a los docentes con mayor tiempo de experiencia, al interior del total de las instituciones analizadas, fue medio-bajo (66\% en ambos grupos de instituciones estudiadas).

Los datos además nos muestran un contexto donde los directivos docentes de instituciones categorizadas en este trabajo como innovadoras en TIC fueron los que mejor valoración presentaron (entre 55\% y 64\%), en torno al empleo de la experimentación y el aprendizaje desde el error y la innovación en las prácticas educativas llevadas a cabo al interior de los centros educativos analizados, así como en la percepción de los directivos docentes alrededor del interés por el uso de las TIC en los profesores de mayor edad y en la disposición general observada al interior de este colectivo, en brindar parte de su tiempo libre al desarrollo de proyectos institucionales mediados por las TIC. Mientras 
que al interior de las instituciones no innovadoras (sin experiencias TIC registradas y sistematizadas) los niveles de percepción aquí expuestos tendieron a ser peores (mediobajo), en la mayoría de los casos estudiados (entre el $53 \%$ y $56 \%$ ).

Cuando hablamos de estrategia de integración de las TIC en los procesos de enseñanzaaprendizaje al interior de las instituciones analizadas, los datos recolectados nos permiten ver cómo, sin importar el tipo de institución abordada, la promoción de incentivos a los docentes no es una estrategia aplicable en la mayoría de los casos (en menos del $20 \%$ de los casos analizados). Así mismo, apreciamos un mayor porcentaje general de centros educativos considerados innovadores (58\%) que aplican una mayor diversidad de estrategias afines a lo aquí expuesto, a saber: la generación de espacios de reflexión sobre el uso de las TIC (la segunda más empleada, por el 68\% de casos relacionados con este grupo); facilitar el acceso a equipos TIC a docentes y estudiantes (la más empleada, en el $88 \%$ de los casos aquí considerados) y otros miembros de la comunidad educativa (ubicada en el sexto lugar, con el 43\%); la gestión de recursos para asegurar el mantenimiento y actualización de los equipos (la cuarta más empleada, con el 56\%); el reconocimiento de las experiencias significativas en el uso educativo de las TIC (nuestra variable dependiente de referencia en este trabajo, ubicada en el quinto puesto de las estrategias más empleadas, con el 48\%); la promoción de la participación del cuerpo docente en eventos, concursos o convocatorias afines al tema aquí propuesto (la tercera más empleada, con el 62\%), así como en la vinculación de los padres y madres de familia en el aprovechamiento de las TIC a nivel institucional (en el último lugar, con el 41\%). En otras palabras, además de observarse como las principales estrategias empleadas en las instituciones innovadoras se enmarcan en facilitar el acceso a la infraestructura TIC a docentes y estudiantes y en el fomento de escenarios reflexivos, tanto fuera como dentro de los espacios institucionales, también se puede ver que, aunque en menor proporción, también se están aplicando estrategias orientadas a fortalecer la relación con otros actores vinculados a la comunidad educativa y a la promoción de escenarios de enseñanza-aprendizaje donde las experiencias significativas cobran especial protagonismo.

Lo expuesto en el párrafo anterior difiere cuando abordamos lo observado en el caso de las instituciones no innovadoras, ya que las estrategias de integración de las TIC tienden a ser más limitadas y abocadas principalmente a facilitar el acceso a equipos TIC a docentes y estudiantes (84\%); la promoción de la participación del cuerpo docente en eventos, concursos o convocatorias afines al tema aquí propuesto $(57 \%)$ y generar espacios de reflexión sobre el uso de las TIC (51\%); y en la gestión de recursos para asegurar el mantenimiento y actualización de los equipos (49\%); mientras que el resto de estrategias son empleadas por un porcentaje menor al $40 \%$ de los casos analizados.

En cuanto a la integración de las TIC en el manual de convivencia, presente en todas las instituciones educativas de Colombia, logramos ver, al menos en la muestra tomada para el abordaje del tema propuesto, que el principal aspecto tenido en cuenta es el cuidado de equipo (más del 85\%), mientras que el uso seguro y respeto de terceros en internet se consideran en menor porcentaje en las instituciones aquí estudiadas (entre 56\% y 65\% en el caso de las instituciones innovadoras y $53 \%$ y $44 \%$ en el caso de las instituciones no innovadoras). Lo que quizás resulta verdaderamente preocupante es la ausencia reconocida por la mayoría de los directivos encuestados de la inclusión de aspectos relacionados con el respeto a los derechos de autor y la formación de ciudadanía digital (menos del 35\% en ambos grupos), aspectos que guardan estrecha relación con la labor impartida por los 
docentes en el aula, sobre todo si tenemos en cuenta que el uso de material proveniente de internet para la construcción de contenidos educativos y el aprendizaje de las normas sociales y de actuación (derechos y deberes ciudadanos en la Red), intrínsecas también en los escenarios digitales, no está siendo considerado en documentos que marcan el devenir de la labor realizada desde las instituciones educativas aquí estudiadas.

Por último, podemos dar cuenta de la percepción dada por los directivos docentes encuestados en torno al tipo de actividades mediadas por las TIC realizadas por sus docentes. Lo anterior nos permite remarcar dos perfiles de docentes:

A nivel de las instituciones innovadoras

- Cuentan con una mayor sistematización y socialización de las innovaciones TIC, llevadas a cabo por estos $(75 \%)$.

- Participan en algún programa nacional de adquisición y aprovechamiento de las TIC $(73 \%)$.

- Hacen parte de instituciones que han desarrollado actividades de alfabetización digital $(71 \%)$.

- Hacen parte de instituciones que han contado en los últimos años con soporte técnico para la implementación de nuevos recursos TIC, para el desarrollo de sus actividades de enseñanza-aprendizaje (68\%).

- Hacen parte de instituciones que han poseído algún convenio de colaboración con actores públicos y privados, orientados a la implementación de las TIC (58\%).

- $\quad$ Poseen algún blog o herramienta 2.0 (58\%).

- Hacen parte de instituciones que han usado algún software educativo, orientado al aprovechamiento formativo de las TIC de sus estudiantes (56\%).

- Han desarrollado alguna actividad de investigación-acción, orientada al tema propuesto $(54 \%)$.

A nivel de las instituciones no innovadoras:

- Participan en algún programa nacional de adquisición y aprovechamiento de las TIC (58\%).

- Hacen parte de instituciones que han desarrollado actividades de alfabetización digital $(70 \%)$.

- Hacen parte de instituciones que han contado en los últimos años con soporte técnico en la implementación de nuevos recursos TIC, para el desarrollo de sus actividades de enseñanza-aprendizaje (50\%).

\subsection{FACTORES QUE INCIDEN EN LA APARICIÓN DE INSTITUCIONES INNOVADORAS EN TIC}

La Tabla 2 nos muestra cómo, desde el punto de vista de la auto-percepción observada por los directivos encuestados de las 172 instituciones educativas consideradas en este estudio, las variables que inciden significativamente en la aparición de instituciones innovadoras en TIC (con presencia de experiencias TIC registradas y sistematizadas) están relacionadas con

- La principal fuente de recursos que ha dotado de equipamiento tecnológico ha sido la Institución Educativa y su comunidad. De acuerdo con el modelo obtenido, la 
probabilidad de que una institución educativa genere experiencias de innovación con base en TIC aumenta en aquellas instituciones $(\operatorname{Exp}(B)=5,623)$ en las cuales la institución y actores como las asociaciones de padres de familia o su entorno comunitario han participado de la consecución de recursos para la dotación de infraestructura TIC, frente a aquellas cuya principal fuente de recursos para este fin han sido los aportes del estado en los distintos niveles de administración educativa.

- La percepción de cambios positivos derivados del uso de las TIC en la planeación institucional. De acuerdo a lo expuesto en la Tabla 2, la probabilidad de la aparición de experiencias innovadoras aumenta $(\operatorname{Exp}(B)=8,778)$ cuando existen altos niveles de acuerdo entre los directivos acerca de la existencia de cambios positivos derivados del uso de las TIC en actividades de planeación institucional, si lo comparamos con aquellos que se ubican en niveles de acuerdo medio y bajo.

- La existencia de proyectos que ayuden a los miembros de la comunidad educativa a adquirir habilidades de organización comprensión, evaluación y análisis de información a través de las TIC. De acuerdo a los datos obtenidos del modelo, es más probable encontrar innovaciones educativas TIC $(\operatorname{Exp}(B)=4,589)$ en aquellas instituciones en las cuales se desarrollan este tipo de proyectos.

- Los docentes con mayor tiempo en su institución se muestran más dispuestos al uso educativo de las TIC. Sobre este punto, la información de la Tabla 2 muestra que la probabilidad de la presencia de innovaciones con base en las TIC en las escuelas se eleva $(\operatorname{Exp}(B)=3,427)$ cuando los directivos de la institución consideran que los docentes con mayor antigüedad muestran una actitud positiva frente al uso de las TIC como herramienta de enseñanza-aprendizaje.

- Formar parte de programas nacionales de adquisición y aprovechamiento de las TIC, al observarse en la Tabla 2 cómo la probabilidad de la generación de innovación aumenta $(\operatorname{Exp}(B)=5,854)$ en aquellas escuelas que participan de dichos programas.

- El reconocimiento de los docentes del aprovechamiento que hacen de las TIC en su IE, bien sea a nivel local, regional o nacional. En aquellas instituciones que cuentan con reconocimientos de este tipo la probabilidad de generar innovación con base en las TIC resultó ser más alta $(\operatorname{Exp}(B)=4,518)$.

- La aplicación de software educativo orientado al aprovechamiento de las TIC. La presencia de este tipo de recursos aumenta la probabilidad de la existencia de experiencias significativas o innovadoras en materia educativa en la muestra de casos seleccionada, mediadas por las TIC $(\operatorname{Exp}(B)=3,863)$. 
Tabla 2. Factores determinantes en la aparición de instituciones innovadoras en TIC

\begin{tabular}{|l|c|c|c|c|c|c|}
\hline \multicolumn{1}{|c|}{ Variables en la ecuación } & B & Error estándar & Wald & gl & Sig. & Exp(B) \\
\hline $\begin{array}{l}\text { Principal fuente de recursos que ha } \\
\text { dotado de equipamiento tecnológi- } \\
\text { co: La Institución Educativa y su } \\
\text { comunidad }\end{array}$ & 1,727 & 0,528 & 10,702 & 1 & 0,001 & 5,623 \\
\hline $\begin{array}{l}\text { Percepción de cambios positivos } \\
\text { derivados del Uso de las TIC en la } \\
\text { Planeación institucional }\end{array}$ & 2,172 & 0,852 & 6,504 & 1 & 0,011 & 8,778 \\
\hline $\begin{array}{l}\text { Existen proyectos que ayudan a los } \\
\text { miembros de la comunidad educati- } \\
\text { va a adquirir habilidades de organi- } \\
\text { zación comprensión, evaluación y } \\
\text { análisis de información a través de } \\
\text { las TIC }\end{array}$ & 1,524 & 0,447 & 11,613 & 1 & 0,001 & 4,589 \\
\hline $\begin{array}{l}\text { Los docentes con mayor tiempo en } \\
\text { su institución se muestran más dis- } \\
\text { puestos al uso educativo de las TIC }\end{array}$ & 1,232 & 0,461 & 7,151 & 1 & 0,007 & 3,427 \\
\hline $\begin{array}{l}\text { Se es parte de algún programa na- } \\
\text { cional de adquisición y aprovecha- } \\
\text { miento de las TIC }\end{array}$ & 1,767 & 0,480 & 13,566 & 1 & 0,000 & 5,854 \\
\hline $\begin{array}{l}\text { Ha sido beneficiario de algún reco- } \\
\text { nocimiento a nivel local, regional, } \\
\text { nacional o internacional desde el } \\
\text { punto de vista del aprovechamiento } \\
\text { de las TIC en su IE }\end{array}$ & 1,508 & 0,471 & 10,232 & 1 & 0,001 & 4,518 \\
\hline $\begin{array}{l}\text { Se ha aplicado algún software edu- } \\
\text { cativo orientado al aprovechamien- } \\
\text { to de las TIC en su IE }\end{array}$ & 1,351 & 0,436 & 9,599 & 1 & 0,002 & 3,863 \\
\hline Constante & $-3,942$ & 0,617 & 40,815 & 1 & 0,000 & 0,019 \\
\hline
\end{tabular}

Nota: $-2 \log$ de la verosimilitud=143,844; R cuadrado de Cox y Snell=0,420; R cuadrado de Nagelkerke=0,561. Solo se exponen en esta tabla las variables introducidas en el modelo por el método de razón de verosimilitudes.

En cuanto al nivel de explicación del modelo expuesto en la Tabla 2 (el $\mathrm{R}^{2}$ de Nagelkerke), nos permite ver, al menos en el caso estudiado (instituciones educativas oficiales), que el conjunto de variables determinantes en torno al tema propuesto logra explicar un estimado del $56,1 \%$ de la variabilidad en la existencia de innovaciones con el uso de las TIC.

Finalmente, la Tabla 3 indica que el nivel de predicción del modelo de explicación en torno a la aparición de instituciones innovadoras en TIC es del 84,3\%. Por tanto, el 
modelo generado, a partir de la variable dependiente tomada en consideración para el desarrollo de este trabajo, presenta un nivel de predicción alto, mientras el nivel de predicción de las instituciones no innovadoras es mayor $(85,9 \%)$ en comparación con el caso de las instituciones innovadoras $(82,5 \%)$. Estos datos dan cuenta de la solidez del constructo tomado en consideración para la identificación de las variables establecidas para la medición aquí expuesta, así como su capacidad de identificar los factores claves, alrededor del tema propuesto.

Tabla 3. Nivel de predicción del modelo de explicación elaborado

\begin{tabular}{|c|c|c|c|c|}
\hline \multirow{3}{*}{ Observado } & \multicolumn{4}{|c|}{ Pronosticado } \\
\hline & & \multicolumn{2}{|c|}{$\begin{array}{c}\text { Existe Experiencia TIC } \\
\text { Sistematizada Socializada }\end{array}$} & \multirow{2}{*}{$\begin{array}{l}\text { Porcentaje } \\
\text { correcto }\end{array}$} \\
\hline & & No & Sí & \\
\hline \multirow{2}{*}{$\begin{array}{l}\text { Existe experiencia TIC } \\
\text { sistematizada socializada }\end{array}$} & No & 79 & 13 & 85,9 \\
\hline & Sí & 14 & 66 & 82,5 \\
\hline \multicolumn{2}{|l|}{ Porcentaje global } & & & 84,3 \\
\hline
\end{tabular}

Fuente: elaborado por los autores.

\section{CONCLUSIONES}

Los datos expuestos en este trabajo no solo permiten reafirmar aspectos destacados por estudios realizados por autores como Ananiadou y Claro (2009) y Area (2010), sobre la importancia que tiene la innovación, tanto a nivel institucional como en el desarrollo profesional de los docentes y en los procesos de enseñanza-aprendizaje aplicados por docentes y estudiantes. También permiten diferenciar los rasgos generales, percepciones y estrategias orientadas a la integración efectiva de las TIC en los escenarios educativos que fueron enmarcados en este trabajo como innovadores con las TIC y aquellos que no fueron categorizados como tales en torno al estudio de caso aquí realizado.

El contexto general observado, más allá de diferencias estructurales entre instituciones analizadas, apunta a resaltar, principalmente, las diferencias observadas en ambos grupos de centros educativos en relación con las formas sobre cómo han venido trascendiendo y aplicando, a nivel práctico-institucional, la alta percepción observada en torno a la búsqueda e implementación de acciones y estrategias orientadas a favorecer la integración de las TIC al interior de estos contextos. Hecho que nos permite, en los términos destacados por Papanastasiou y Ferdig (2006), Claro (2010) y IIPE-UNESCO (2006), demarcar dos tipos de instituciones al momento de asumir el cómo y para qué hacer uso de las TIC, en aras a sacar el máximo provecho de estas, tanto a nivel comunicativo como educativo: 
- Unas instituciones ubicadas más entre el momento internet y actual destacado por la IIPE-UNESCO (2006), con un rol más activo en la búsqueda de mecanismos que aseguren el mayor aprovechamiento de las TIC para el desarrollo de sus labores de enseñanza-aprendizaje, desde los diferentes agentes educativos que las integran.

- Unas instituciones ubicadas más en el momento informático señalado por la IIPE-UNESCO (2006), con un rol más pasivo, donde el liderazgo asumido por los agentes educativos no logra trascender las percepciones favorables alrededor del potencial impacto de las TIC, a favor de un entorno más positivo de realización del tema aquí abordado: la innovación educativa mediada por TIC.

Bajo lo antes expuesto, al menos en el caso del estudio tenido en cuenta en este artículo, pareciera estar dominado por el segundo grupo de instituciones educativas, lo que haría posible encontrarnos en un contexto donde lo expuesto por Cox et al. (2003) sería aún muy difícil de concretar o al menos generalizar a nivel de la región Caribe colombiana, con lo que se reafirmaría lo dicho por Fullan (2007), así como Montero y Gewerc (2010), García-Valcárcel y Tejedor (2011), Ling, Woo y Lim (2013), Arias y Cristia (2014), en torno a avanzar más allá del reconocimiento de las potencialidades de las TIC en el desarrollo de la vida escolar o el discurso de la innovación, para pasar a un contexto donde se transforme la cultura institucional de un alto número de instituciones educativas analizadas en materia TIC, a través del fomento de una planificación estratégica orientada a una mayor articulación de los recursos y dispositivos tecnológicos dispuestos en la actualidad en los currículos y en los demás procesos educativos, pedagógicos y administrativos, desde una contextualización de lo aquí señalado a la realidad inherente de cada uno de los escenarios de enseñanza.

Lo observado en los datos también nos lleva a reafirmar lo expuesto por autores como Craig et al. (2011), Sang et al. (2011), Fariña y Sosa, 2011, Ramírez et al. (2012), Area, Gutiérrez y Vidal (2012), Arias y Cristia, 2014 o Said-Hung et al. (2015), en cuanto a la necesidad de favorecer un contexto donde se mejore el compromiso y actitud positiva no solo de los docentes y estudiantes, sino del resto de gestores educativos (directivos docentes y coordinadores académicos, por ejemplo). Ello en vista del importante liderazgo que cumplen todos en la promoción de un entorno donde se implemente una mayor diversidad de estrategias orientadas a la integración favorable de las TIC en los contextos de enseñanza-aprendizaje observados en el caso de las instituciones educativas categorizadas aquí como innovadoras. Así se reafirmaría lo expuesto por Beraún (2011) y Said-Hung et al. (2015) en torno al reconocimiento del importante rol que cumple el liderazgo de los docentes, pero también de los directivos docentes o rectores encuestados, por ejemplo, alrededor del tema aquí tratado.

El modelo explicativo expuesto en este trabajo también nos marca un conjunto de acciones que se deberían tener en cuenta, tanto a nivel general como al interior de las instituciones vinculadas al estudio de caso expuesto en este trabajo, a nuestro modo de ver: la necesidad de impulsar una mayor participación y compromiso en el proceso de integración de las TIC por parte de las instituciones educativas y comunidad donde se encuentra desarrollando su actividad formativa; fomentar la aplicación de estrategias que aseguren la integración de las tecnologías en los procesos comunicativos y evaluaciones institucionales, pero sobre todo en la planificación al interior de cada centro educativo; promover mecanismos que ayuden a atraer, de forma más activa, a los docentes de mayor tiempo al interior de las instituciones educativas, de forma que asuman el rol clave que tienen a favor de la promoción de una nueva generación de miembros de este colectivo, 
integrados tecnológicamente; garantizar y fortalecer los mecanismos de reconocimientos institucionales y públicos de los docentes y entidades educativas innovadoras; asî como la presencia y uso activo de recursos educativos digitales que faciliten un mayor aprovechamiento de las TIC en los centros educativos analizados. Todo ello dirigido, en especial, a garantizar que los docentes que ingresen o se encuentren en los centros educativos analizados apoyen la transformación de estas instituciones, a favor de la promoción de experiencias de innovación educativa en TIC en ellas, a través de la revisión de los diferentes programas y estrategias puestos en marcha para la dinamización de la política pública actualmente existente en el país, la cual incide con el caso de estudio aquí tenido en cuenta.

\section{REFERENCIAS BIBLIOGRÁFICAS}

Ait, K., Rannikmäe, M., Soobard, R., Reiska, P., \& Holbrook, J. (2015). Students' Self-Efficacy and Values Based on 21st Century Vision of Scientific Literacy - A Pilot Study. Procedia - Social and Behavioral Sciences, 177, 491-495.

Ananiadou, K., \& Claro, M. (2009). 21st Century Skills and Competences for New Millennium Learners in OECD Countries. OECD Education Working Papers, 41. doi:10.1787/218525261154

Area, M. (2010). El proceso de integración y uso pedagógico de las TIC en los centros educativos. Un estudio de caso. Revista de Educación, 352, 77-97.

Area, M., Gutiérrez, A., \& Vidal, F. (2012). Alfabetización digital y competencias informacionales. Madrid: Fundación Telefónica.

Arias, E., \& J. Cristia. (2014). El BID y la tecnología para mejorar el aprendizaje: ¿Cómo promover programas efectivos? Washington: Banco Interamericano de Desarrollo.

Benavides, F., \& Pedró, F. (2007) Políticas educativas sobre nuevas tecnologías en los países Iberoamericanos. Revista Iberoamericana de Educación, 45, 19-69.

Beraún, L. (2011). Teoría de los rasgos por liderazgo para generar capacidades. Facultad de Ciencias Administrativas de la Universidad Nacional, Huánuco, Perú. Recuperado de http:// www.scribd.com/doc/56014709/Liderazgo-Para-Generar-Capacidades.

Binkley, M., Erstad, O., Herman, J., Raizen, S., Ripley, M., Miller-Ricci, M., \& Rumble, M. (2012). Defining Twenty-First Century Skills. In B. McGaw \& E. Care (Eds.), Assessment and Teaching of 21st Century Skills (pp. 17-66). New York, NY: Springer.

Boza, Á., Tirado, R., \& Guzmán-Franco, M. (2010). Creencias del profesorado sobre el significado de la tecnología en la enseñanza: influencia para su inserción en los centros docentes andaluces. Relieve, 16(1), 1-24.

Claro, M. (2010). Impacto de las TIC en los aprendizajes de los estudiantes. Estado del Arte. Santiago de Chile: CEPAL.

Claro, M., Preiss, D., San Martín, E., Jara, I., Hinostroza, E., Valenzuela, S., Cortes, F., \& Nussbaum, M. (2012). Assessment of 21st century ICT skills in Chile: Test design and results from high school level students. Computers \& Education, 59, 1042-1053.

Coscollola, M., \& Marquès, P. (2011). Aulas 2.0 y uso de las TIC en la práctica docente. Revista Comunicar, 37(XIX), 169-175.

Cox, M., Abbott, C., Webb, M., Blakeley, B., Beauchamp, T., \& Rhodes, V. (2003). ICT and attainment: A review of the research literature ICT in Schools. Research and Evaluation Series, 17. Recuperado de http://webarchive.nationalarchives.gov.uk/20130401151715/http://www. education.gov.uk/publications/eOrderingDownload/ICT\%20and\%20attainment.pdf

Craig, J., Ault, M., \& Niileksela, C. (2011). The Influence of Technology Rich Learning Environments: A Classroom-based Observational Study. In Proceedings of Society for Information Technology 
\& Teacher Education International Conference 2011 (pp. 4304-4311). Chesapeake, VA: AACE. Daniels, K. (2015). Los adolescentes ya no son responsables del aprendizaje: declaraciones a la web de recursos educativos. Recuperada de http://blog.tiching.com/kristin-daniels-los-docentes-yano-son-responsables-del-aprendizaje/\#.VRfUV_cENxg.twitter.

Fariña, F., \& Sosa, J. (2011). Reflexión y mejora en la práctica docente: narración de una experiencia en el área de tecnología. Qurriculum, 24, 118-142.

Fullan, M. (2007). The new meaning of educational change (4th ed.). New York: Teachers College Press.

García-Valcárcel, A., \& Tejedor, F. (2011). Características y valoración de los escenarios de enseñanzaaprendizaje con TIC en el ámbito universitario. En R. Roig y M. Fiorucci (Coords.), Claves para la investigación en innovación y calidad educativas, la integración de las tecnologías de la información y la comunicación y la interculturalidad en las aulas (pp. 179-192). Madrid: Editorial Marfil.

IIPE-Unesco. (2006). La integración de las Tecnologías de la Información y de la Comunicación en los Sistemas Educativos. Estado del arte y orientaciones estratégicas para la definición de políticas educativas en el sector. Buenos Aires: Autor.

Ling, J., Woo, H., \& Lim, W. (2013). Understanding the relationship between Singapore preservice teachers' ICT course experiences and technological pedagogical content knowledge (TPACK) through ICT course evaluation. Educational Assessment, Evaluation and Accountability, 25(4), 321-339.

Montero, L., \& Gewerc, A. (2010). De la innovación deseada a la innovación posible. Escuelas alteradas por las TIC. Profesorado. Revista de currículum y formación del profesorado, 14(1), 303-318.

Oviedo, C., \& Campo, A. (2005). Aproximación al uso del coeficiente Alfa de Cronbach. Revista Colombiana de Psiquiatría, XXXIV(4), 572-580.

Papanastasiou, E., \& Ferdig, R. (2006). Computer Use and Mathematical Literacy: An Analysis of Existing and Potential Relationships. Journal of Computers in Mathematics and Science Teaching, 25(4), 361-371.

Peñafiel, F. (2000). Las nuevas tecnologías y la educación de alumnos con necesidades educativas especiales en los umbrales del siglo XXI. Recuperado de http://www.quadernsdigitals.net/ index.php?accionMenu=hemeroteca.VisualizaArticuloIU.visualiza\&articulo_id=2632.

Ramírez, E., Cañedo, I., \& Clemente, M. (2012). Las actitudes y creencias de los profesores de secundaria sobre el uso de Internet en sus clases. Comunicar, 38(XIX), 147-1551. doi:10.3916/ C38-2012-03-06

Said-Hung, E., Iriarte, F., Valencia, J., Borja, M., Ordóñez, M., Arellano, W., . . Román, G. (2015). Hacia el fomento de las TIC en el sector educativo en Colombia. Colombia: Ediciones Uninorte.

Sang, G., Valcke, M., Van Braak, J., Tondeur, J., \& Zhu, CH. (2011). Predicting ICT integration into classroom teaching in Chinese primary schools: exploring the complex interplay of teacherrelated variables. Journal of Computer Assisted Learning, 27, 160-172. doi:10.1111/j.13652729.2010.00383.x

Sunkel, G., Trucco, D., \& Espejo, A. (2013). La Integración de las Tecnologías Digitales en las Escuelas de América Latina y el Caribe: Una Mirada Multidimensional. Santiago de Chile: Naciones Unidas.

Unesco. (2013). Uso de TICs en Educación en América Latina y el Caribe: Análisis Regional de la Integración de las TICs en la Educación y de la Aptitud Digital (e-readiness). Montreal: Autor.

Vanderlinde, R., \& Van Braak, J. (2011). A New ICT Curriculum for Primary Education in Flanders: Defining and Predicting Teachers' Perceptions of Innovation Attributes. Educational Technology, 14(2), 124-135.

Vera, M. (2004). La enseñanza-aprendizaje virtual: principios para un nuevo paradigma de instrucción y aprendizaje. En M. I. Vera y D. Pérez (Eds.), La formación de la ciudadanía: Las TICs y los nuevos problemas (pp. 57-64). Alicante: AUPDCS. 
